\title{
Dermatofibrosarcoma Protuberans in the Palm of a 3-Year-Old Child
}

\author{
Omer Faruk Kumbuloglu${ }^{1}$, Fatih Barishan ${ }^{2}$, Fevziye Kabukcuoglu ${ }^{3}$, Haci Mustafa Ozdemir ${ }^{2}$ \\ 1 Department of Orthopaedics and Traumatology, Hand Surgery Division, Sisli Hamidiye Etfal Training and Research Hospital, \\ Istanbul, Turkey \\ 2 Department of Orthopaedics and Traumatology, Sisli Hamidiye Etfal Training and Research Hospital, Istanbul, Turkey \\ 3 Department of Pathology, Istanbul Sisli Hamidiye Etfal Application and Research Center, University of Health Sciences, Istanbul, Turkey
}

Key words: pediatric dermatofibrosarcoma protuberans, handpalm masses, Mohs micorgraphic surgery, imatinib

Citation: Kumbuloglu OF, Barishan F, Kabukcuoglu F, Ozdemir HM. Dermatofibrosarcoma protuberans in the palm of a 3-year-old child. Dermatol Pract Concept. 2022;12(1):e2022015. DOI: https://doi.org/10.5826/dpc.1201a15

Accepted: May 5, 2021; Published: January 2022

Copyright: $\odot 2022$ Kumbuloglu et al. This is an open-access article distributed under the terms of the Creative Commons Attribution-NonCommercial License (BY-NC-4.0), https://creativecommons.org/licenses/by-nc/4.0/, which permits unrestricted noncommercial use, distribution, and reproduction in any medium, provided the original authors and source are credited.

Funding: None.

Competing interests: None.

Authorship: All authors have contributed significantly to this publication.

Corresponding author: Fatih Barishan, MD, Department of Orthopaedics and Traumatology, Sisli Hamidiye Etfal Training and Research Hospital, Istanbul, Turkey. E-mail: drbarishan@hotmail.com

\section{Introduction}

Dermatofibrosarcoma protuberans (DFSP) is a low-grade, locally invasive tumor that originates from cutaneous tissues. DFSP is the second most frequent skin sarcoma and is generally diagnosed between the ages of 30 and 50. It is mostly localized in the trunk, proximal extremities, or in the head and neck region [1]. There are a few reports showing DFSP in the dorsum of the hand of pediatric cases [2]. In this study, a case is presented of a 3-year-old boy that underwent 3 operations for DFSP in the palm of the hand.

\section{Case Presentation}

A 3-year-old male child presented to our clinic with a mass lesion at the level of the second metacarpophalangeal joint in the palm of the right hand. Two months before, the patient underwent surgical excision on the palm of his right hand at another clinic. The histological diagnosis was reported as lipofibromatosis at that time. Physical examination revealed a $1.5 \mathrm{~cm} \times 2 \mathrm{~cm}$ mass in the same region (Figure $1 \mathrm{~A}$ ).

In the second surgical excision of the patient, the mass lesion was excised together with the overlying skin. The defective area that occurred at the excision site was closed with a full-thickness skin graft obtained from the right inguinal area. The histological examination showed a spindle cell lesion that started just below the epidermis and reaching the dermis and subcutaneous fat tissue, with short bundles crossing each other and continued beyond the surgical border. Marked atypia or mitotic activity was not observed. Immunohistochemical studies revealed widespread CD34 staining. The focal staining properties were observed using caldesmon and Alpha Smooth Muscle Actin stains. Desmin stain, HMB45, pancytokeratin, CD31, and calponin were all negative. The 


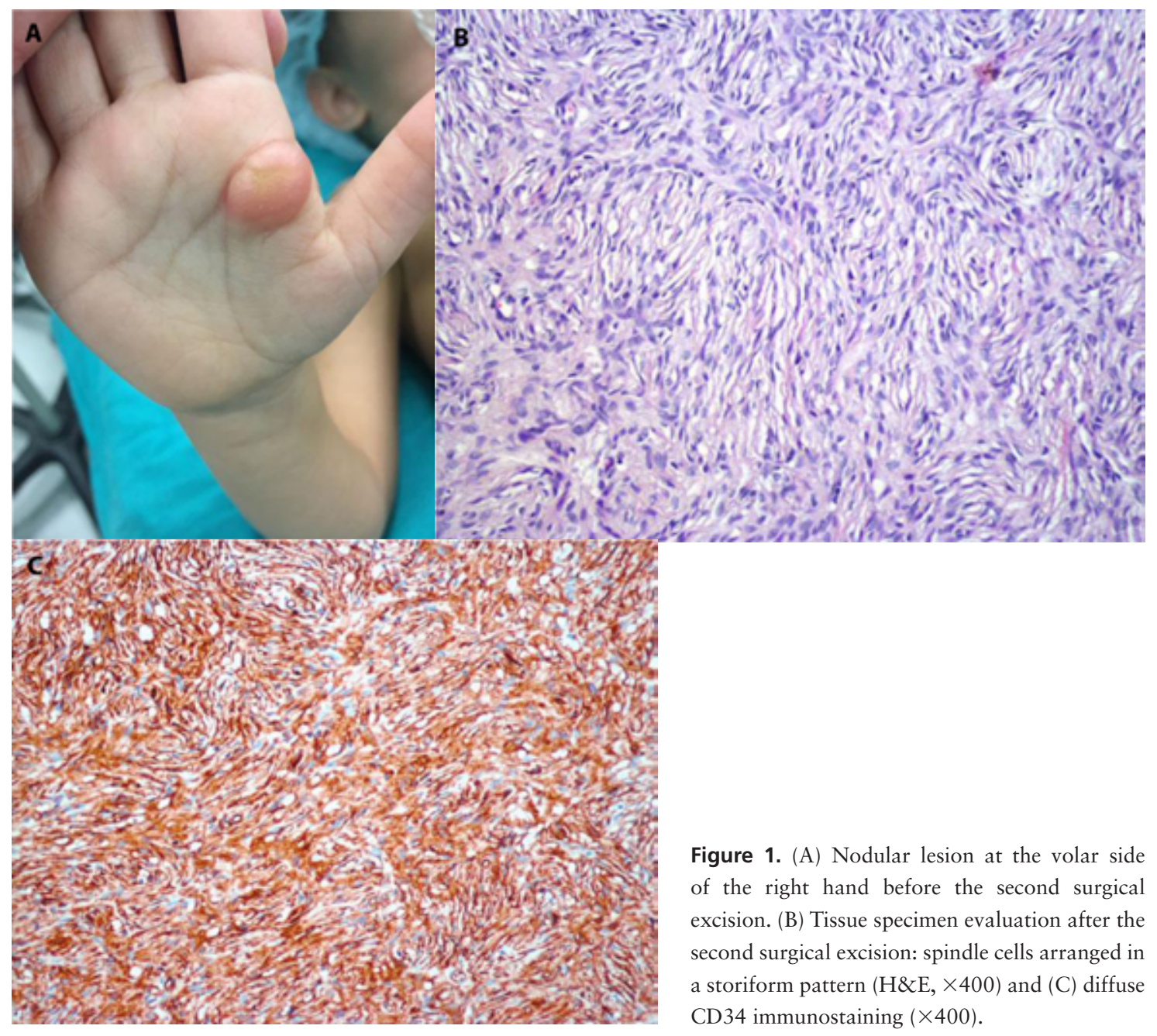

Ki67 proliferation index was approximately 2-5\% and the lesion was diagnosed as DFSP (Figure 1, B and C).

The patient was reevaluated following the histological diagnosis of DFSP and a subsequent chest X-ray did not show metastasis. Physical examination did not show any sign of lymphadenopathy. The third surgical excision was planned for the patient.

In the third surgical excision Mohs micrographic surgery was used. The surgical excision site was mapped with the tumor in the middle (Figure 2, A and B). The whole epidermal tissue, dermal tissue, first lumbrical muscle, and common palmar digital nerve were excised together with a tissue margin of $1 \mathrm{~cm}$ (Figure 2C). Since intraoperative frozen examination was reported as negative for the surgical border, a $4 \mathrm{~cm} \times 3 \mathrm{~cm}$ wound site was left open and covered with surgical dressing, and a plaster was applied. The wound healed with a secondary closure in 6 weeks. There was no

need for radiotherapy or imatinib treatment. There was no sign of recurrence at the end of the first postoperative year (Figure 2, D-F).

\section{Conclusions}

A diagnosis of DFSP is made upon clinical examination and histological evaluation of the patient and is often difficult due the frequently asymptomatic and slow progression. Histological diagnosis cannot be made with routine staining. Clinicians should consider DFSP in the differential diagnosis for pediatric palmar cutaneous masses; otherwise, a delay in diagnosis may cause a loss of hand function.

Informed consent: Written informed consent for publication of clinical details and clinical images was obtained from the patient. 


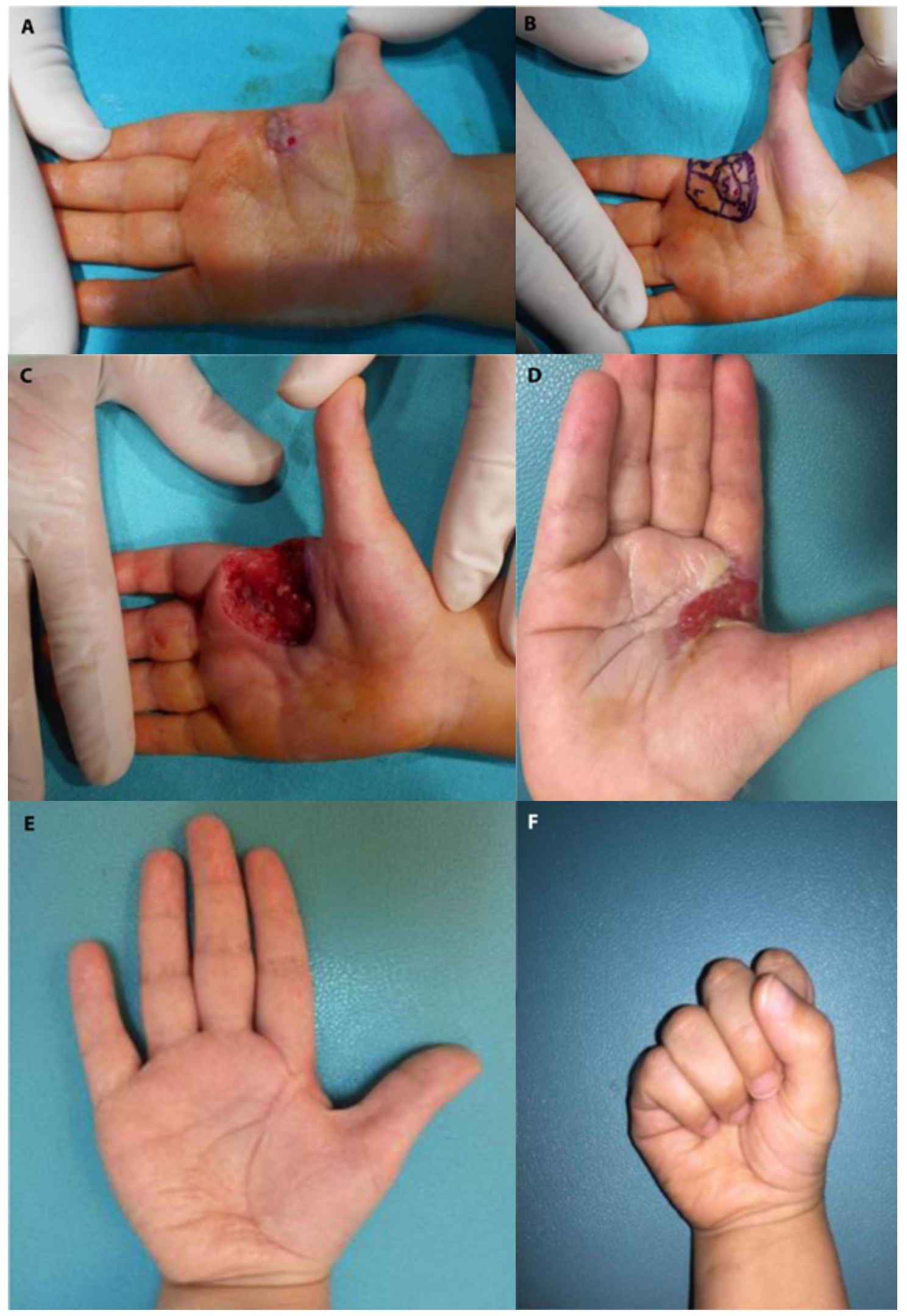

Figure 2. (A) Appearance of the hand before Mohs micrographic surgery. (B) Tumor mapping, placing the lesion in the center. (C) After Mohs micrographic surgery. (D) Wound healing process following Mohs micrographic surgery 4 weeks postoperatively. (E, F) Appearance of the hand following Mohs micrographic surgery 1 year postoperatively with good function and no contracture development. 


\section{References}

1. Shah KK, McHugh JB, Folpe AL, Patel RM. Dermatofibrosarcoma protuberans of distal extremities and acral sites: a clinicopathologic analysis of 27 cases. Am J Surg Pathol. 2018;42(3):413-419. DOI: 10.1097/PAS.0000000000000998. PMID: 29240584.
2. Tsai YJ, Lin PY, Chew KY, Chiang YC. Dermatofibrosarcoma protuberans in children and adolescents: Clinical presentation, histology, treatment, and review of the literature. J Plast Reconstr Aesthet Surg. 2014;67(9):1222-1229. DOI: 10.1016/j. bjps.2014.05.031. PMID: 24973861. 